\section{PSYCAUSE}

Revue scientifique étudiante de

l'École de psychologie de l'Université Laval
UNIVERSITÉ LAVAL

Faculté des sciences sociales École de psychologie

SEPTEMBRE 2018 - VOL. $8 \mathrm{~N}^{\circ} 2$

\title{
PARTICULARITÉS DES MĖRES RECRUTÉES DANS UN PROGRAMME DE SOUTIEN À LA PARENTALITÉ
}

Florence FILION ${ }^{1, *}$, Lisa-Marie GAGNÉ1, Maude LACHAPELLE ${ }^{1} \&$ Marie-Hélène GAGNÉ ${ }^{1}$

1 École de psychologie, Université Laval, Québec, Canada

* florence.filion.1@ulaval.ca

\section{Pour citer l'article}

Filion, F., Gagné, L.-M., Lachapelle, M., \& Gagné, M.-H. (2018). Particularités des mères recrutées dans un programme de soutien à la parentalité. Psycause : Revue scientifique étudiante de l'École de psychologie de l'Université Laval, 8(2), 26-29. 


\section{PARTICULARITÉS DES MÈRES RECRUTÉES DANS UN PROGRAMME DE SOUTIEN À LA PARENTALITÉ}

Florence FILION, Lisa-Marie GAGNÉ, Maude LACHAPELLE \& Marie-Hélène GAGNÉ École de psychologie, Université Laval, Québec, Canada

\section{Introduction}

Les programmes de soutien à la parentalité, comme le système d'intervention Triple $P$ Positive Parenting Program, sont des moyens efficaces pour prévenir la maltraitance envers les enfants et diminuer les facteurs de risques associés (Prinz, Sanders, Shapiro, Whitaker \& Lutzker, 2016). Présenté comme une approche de santé publique non stigmatisante, Triple $P$ se segmente en cinq niveaux d'intervention d'intensité croissante et de plus en plus ciblée. Ce programme a récemment été expérimenté au Québec dans deux territoires de centres locaux de services communautaires (CLSC; Gagné, Drapeau \& Charest, 2017). Un défi des programmes de soutien à la parentalité est toutefois de s'assurer qu'ils rejoignent les tranches les plus vulnérables de la population (Shapiro, Prinz \& Sanders, 2010). Ces programmes devraient démontrer leur capacité à rejoindre les jeunes familles de milieux défavorisés, celles monoparentales ou recomposées ainsi que celles dont les parents sont moins confiants et plus stressés et dont les enfants sont plus difficiles, car ces types de familles présentent des facteurs de risque qui ont été associés à la maltraitance (Organisation mondiale de la Santé, 2006). La présente étude a pour but de vérifier si le programme Triple $P$ a su rejoindre ces familles. Spécifiquement, elle déterminera jusqu'à quel point les mères s'engageant dans le système Triple $P$ aux niveaux 3 ou 4 , consistant en des interventions en groupe ou individuelles, présentent des facteurs de risque de maltraitance et si ces caractéristiques les distinguent de la norme de leur territoire. II est attendu que les mères ayant participé au programme présentent en moyenne davantage de facteurs de risque associés à la maltraitance que celles de la population générale.

\section{Méthode}

Cette étude exploite deux ensembles de données recueillies dans le cadre de l'évaluation québécoise du programme Triple $P$. Le premier groupe est composé d'un échantillon de 834 mères ayant participé à une enquête téléphonique, choisies aléatoirement dans la population générale, provenant des territoires de CLSC Mercier-Est Anjou, dans la région de Montréal $(n=417)$ et Orléans dans la région de Québec ( $n=417)$. Le second groupe est composé de 240 mères, provenant des mêmes territoires (Mercier-Est Anjou, $n=112$; Orléans, $n=128$ ), ayant rempli un questionnaire autoadministré lors de leur entrée aux niveaux 3 et 4 de Triple $P$. Par souci de comparaison, seules les mères d'enfants âgés de 6 mois à 8 ans ont été incluses dans les deux groupes.

Le comportement de l'enfant est évalué par les quatre sous-échelles du Strengths and Difficulties Questionnaire (Goodman, 1997). Le sentiment de compétence parentale est mesuré par une question du Parenting Stress Index - short form (Abidin, 1995). Les pratiques parentales positives sont évaluées depuis le score obtenu à la sous-échelle Pratiques éducatives positives de l'Alabama Parenting Questionnaire (Shelton, Frick \& Wooton, 1996).

\section{Résultats et discussion}

Le Tableau 1 présente une description de chacun des groupes au regard des variables dichotomiques ou catégorielles à l'étude ( $n$ et \%), la signification statistique $(p)$ et les tailles d'effet 
$\left(\Phi, \Phi_{C}\right)$ des différences de proportions observées entre les groupes. Les différences de moyennes observées sur le plan des variables continues sont présentées au Tableau 2 ainsi que la signification statistique $(p)$ et les tailles d'effet $\left(d, \eta^{2}\right)$.

Les résultats montrent des différences significatives entre les groupes suggérant que le programme Triple $P$ a été implanté de manière à rejoindre des familles affichant plusieurs facteurs de risque. Les différences les plus marquées se situent sur le plan du comportement des enfants. Les mères qui s'engagent dans Triple $P$ perçoivent leur enfant comme étant beaucoup plus difficile et observent chez lui davantage de comportements agressifs, opposants, hyperactifs et inattentifs, et de troubles affectifs que les mères de la population générale. Leur volonté à rechercher de nouveaux outils pour mieux encadrer les comportements problématiques et pour se sentir plus compétentes pourrait être un incitatif majeur de participation au programme. Le revenu familial et les variables de parentalité distinguent modérément les groupes. Un faible niveau socioéconomique et une situation familiale complexe ont été associés à davantage de stress pouvant engendrer de l'impatience, du découragement et des

\section{Tableau 1}

Description de l'échantillon et différences de proportion entre les groupes clinique et normatif

\begin{tabular}{|c|c|c|c|c|c|c|}
\hline & \multirow{2}{*}{$\begin{array}{c}\text { Groupe } \\
\text { normatif } \\
n=834 \\
n(\%)\end{array}$} & \multirow{2}{*}{$\begin{array}{c}\text { Groupe } \\
\text { clinique } \\
n=240 \\
n(\%)\end{array}$} & \multirow[b]{2}{*}{$p$} & \multicolumn{2}{|c|}{ Taille d'effet } & \multirow{2}{*}{$\begin{array}{c}\text { Éch. total } \\
N(\%)\end{array}$} \\
\hline & & & & $\Phi$ & $\Phi_{C}$ & \\
\hline \multicolumn{7}{|l|}{ Sexe de l'enfant } \\
\hline Garçon & $416(49,9)$ & $150(63,0)$ & $<0,001$ & 0,11 & & $566(52,8)$ \\
\hline Fille & $418(50,1)$ & $88(37,0)$ & & & & $506(47,2)$ \\
\hline \multicolumn{7}{|l|}{ Statut d'emploi } \\
\hline Sans emploi & $140(16,9)$ & $97(40,4)$ & $<0,001$ & $-0,24$ & & $237(22,2)$ \\
\hline Emploi rémunéré & $689(83,1)$ & $143(59,6)$ & & & & $832(77,8)$ \\
\hline \multicolumn{7}{|l|}{ Structure familiale } \\
\hline Biparentale intacte & $697(84,5)$ & $138(57,5)$ & $<0,001$ & & 0,28 & $835(78,4)$ \\
\hline Recomposée & $40(4,8)$ & $26(10,8)$ & & & & $66(6,2)$ \\
\hline Monoparentale & $88(10,7)$ & $76(31,7)$ & & & & $164(15,4)$ \\
\hline \multicolumn{7}{|l|}{ Niveau de scolarité } \\
\hline Secondaire et moins & $88(10,6)$ & $53(22,1)$ & $<0,001$ & & 0,19 & $141(13,2)$ \\
\hline Collégial & $308(37,2)$ & $109(45,4)$ & & & & $417(39,0)$ \\
\hline Universitaire & $433(52,2)$ & $78(32,5)$ & & & & $511(47,8)$ \\
\hline \multicolumn{7}{|c|}{ Sentiment compétence parentale } \\
\hline Pas très bon/a difficultés & $7(0,8)$ & $59(24,6)$ & $<0,001$ & & 0,42 & $66(6,2)$ \\
\hline Aussi bon que moyenne & $393(47,2)$ & $110(45,8)$ & & & & $503(46,9)$ \\
\hline Meilleur que moyenne & $239(28,7)$ & $39(16,3)$ & & & & $278(25,9)$ \\
\hline Très bon parent & $194(23,3)$ & $32(13,3)$ & & & & $226(21,1)$ \\
\hline
\end{tabular}


craintes en plus de contribuer à l'intention des mères à chercher de l'aide (Prinz, 2016). Les mères monoparentales, particulièrement, peuvent se sentir moins outillées, confiantes et soutenues dans leur rôle parental (Waldfogel et coll., 2010). Considérant ces résultats, il est possible de conclure que Triple $P$ a su rejoindre des familles présentant plusieurs facteurs de risque liés à la maltraitance, ce qui en fait un programme prometteur pour contribuer à réduire les disparités sociales en santé et bienêtre des familles. Dans les recherches futures, il serait intéressant de prendre en compte le point de vue des pères et de mesurer si l'implantation de Triple $P$ a réellement permis de réduire les taux de maltraitance dans les territoires concernés.

\section{Références}

Abidin, R. R. (1995). Parenting Stress Index Short Form: Test Manuel. Charlottesville, VA: Pediatric Psychology Press.

Gagné, M.-H., Drapeau, S., \& Charest, É. (2017, juin). Les effets du programme Triple P. Conférence présentée aux $15^{\mathrm{e}}$ Journées annuelles de la recherche sur les jeunes et les familles. De la prévention à la réadaptation à Institut universitaire du Centre de recherche du Centre jeunesse de Québec (CIUSSS de la Capitale-Nationale), Québec.

Goodman, R. (1997). The Strengths and Difficulties Questionnaire: A research note. Journal of Child Psychology and Psychiatry, 38, 581-586. doi:10.1111/j.1469-7610.1997.tb01545.x

Organisation Mondiale de la Santé (2006). Guide sur la prévention de la maltraitance des enfants: Intervenir et produire des données. France: Organisation mondiale de la Santé et International Society for Prevention of Child Abuse and Neglect

\section{Tableau 2}

Description de l'échantillon et différences de moyennes entre les groupes clinique et normatif

\begin{tabular}{lccccc}
\hline & $\begin{array}{c}\text { Groupe } \\
\text { normatif } \\
(n=834)\end{array}$ & $\begin{array}{c}\text { Groupe } \\
\text { clinique } \\
(n=240)\end{array}$ & $p$ & \multicolumn{2}{c}{ Taille d'effet } \\
\hline & $M(E ́-T)$ & $M(E ́-T)$ & & & $\eta^{2}$ \\
\hline Âge de la figure maternelle & $34,49(5,31)$ & $33,90(6,06)$ & 0,144 & - & \\
Âge de l'enfant & $4,91(2,37)$ & $5,37(2,06)$ & 0,003 & $-0,22$ & \\
Revenu & $6,40(2,11)$ & $4,87(2,70)$ & $<0,001$ & 0,60 & \\
Pratiques parentales positives & $4,32(0,42)$ & $4,11(0,53)$ & $<0,001$ & 0,40 & \\
Perception avoir enfant difficile & $2,03(0,65)$ & $2,58(0,58)$ & $<0,001$ & $-0,87$ & \\
Comportement de l'enfant & & & & & \\
Troubles affectifs & $0,29(0,34)$ & $0,67(0,46)$ & $<0,001$ & & 0,14 \\
Troubles de comportement & $0,42(0,36)$ & $0,99(0,45)$ & $<0,001$ & & 0,27 \\
Hyperactivité/inattention & $0,60(0,46)$ & $1,16(0,52)$ & $<0,001$ & & 0,19 \\
Prosocialité & $1,60(0,39)$ & $1,35(0,39)$ & $<0,001$ & & 0,07 \\
\hline
\end{tabular}

Note. Les barèmes d'interprétation pour le $d$ de Cohen (d) sont: $<0,2=$ effet de petite taille; autour de 0,5= effet de moyenne taille; $>0,8=$ effet de grande taille, et ceux pour l'êta-carré partiel $\left(\eta^{2}\right)$ sont: $<0,01=$ effet de petite taille; autour de 0,06 = effet de moyenne taille, $>0,14=$ effet de forte taille.

5 Le revenu est divisé en catégories distinctes, mais a été considéré comme étant une variable continue: 1 . Moins de $15000 \$$, 2. [15 000, 25 000[, 3. [25 000, 35 000[, 4. [35 000, 45 000[, 5. [45 000, 55 000[, 6. [55 000, 65 000[, 7. $[65000,75000[, 8.75000 \$$ et plus. 
Prinz, R. J. (2016). Parenting and family support within a broad child abuse prevention strategy: Child maltreatment prevention can benefit from public health strategies. Child Abuse \& Neglect, 51, 400-406. doi:10.1016/j. chiabu.2015.10.015

Prinz, R. J., Sanders, M. R., Shapiro, C. J., Whitaker, D. J., \& Lutzker, J. R. (2016). Addendum to «Population-based prevention of child maltreatment: The U.S. Triple P system population trial». Prevention Science, 17, 410-416. doi:10.1007/s11121-016-0631-x
Shapiro, C. J., Prinz, R. J., \& Sanders, M. R. (2010). Population-based provider engagement in delivery of evidence-based parenting interventions: challenges and solutions. Journal of Primary Prevention, 31, 223-234. doi:10.1007/s10935-010-0210-z

Shelton, K.K., Frick, P. J., \& Wootton, J.M. (1996). Assessment of parenting practices in families of elementary school-age children. Journal of Clinical Child Psychology, 25, 317-329. doi:10.1207/s15374424jccp2503_8

Waldfogel, J., Craigie, T. A., \& Brooks-Gunn, J. (2010). Fragile families and child wellbeing. Future Child, 20, 87-112. doi:10.1353/ foc. 2010.0002

\section{PROFILS DE RÉPERCUSSIONS SEXUELLES CHEZ DES FEMMES VICTIMES D'AGRESSION SEXUELLE À L'ÂGE ADULTE}

Laurie CÔTÉ, Stéphanie DUCHESNE, Justine MITCHELL, Marie-Pier VAILLANCOURT-MOREL, Stéphane SABOURIN \& Elodie HAMEL École de psychologie, Université Laval, Québec, Canada

\section{Introduction}

L'agression sexuelle à l'âge adulte (ASA) est un acte de nature sexuelle avec ou sans contact direct posé par un individu sur une victime âgée d'au moins 16 ans sans son consentement (Gouvernement du Québec, 2001). De nombreuses séquelles sexuelles sont observées chez les femmes à la suite d'une ASA. En effet, ces femmes rapportent des taux plus élevés de détresse sexuelle (Conoscenti \& McNally, 2006), de compulsion sexuelle (Brown et coll., 2003), d'évitement sexuel (Gidycz \& Kelley, 2016) et de perturbations sexuelles (O'Driscoll \& Flanagan, 2016). Ces femmes vivent également une baisse de satisfaction sexuelle (Offman \& Matheson, 2004) et de la fonction sexuelle (Turchik \& Hassija, 2014). Certaines variables sont reconnues pour avoir une influence sur les répercussions sexuelles à la suite d'une ASA. Comparativement aux femmes célibataires, les femmes mariées sont moins susceptibles d'adopter des comportements sexuels à risque (Mittal et coll., 2013). De plus, les femmes de bas niveau socioéconomique s'engagent davantage dans des relations sexuelles non protégées (Wingood \& DiClemente, 1998). Aussi, Burri, Schweitzer et O'Brien (2014) rapportent que, dans la population générale, de hauts niveaux d'anxiété d'abandon et d'évitement de l'intimité affectent négativement l'orgasme, la satisfaction sexuelle etlefonctionnementsexuel. En ce sens, l'objectif premier de la présente étude est d'identifier les différents profils de répercussions sexuelles chez des femmes victimes d'ASA. L'objectif secondaire est de vérifier si les variables sociodémographiques et la nature de l'attachement amoureux sont associées à l'appartenance à un profil particulier de séquelles sexuelles. 